\title{
A importância da atividade física regular em grupos de sedentários pós pandemia por COVID-19: revisão de literatura
}

The importance of regular physical activity in sedentary subjects post-COVID-19 pandemic: literature review

\author{
La importancia de la actividad física regular en sujetos sedentarios post pandemia COVID-19 \\ revisión de literatura
}

\author{
EIson Nobrega da Silva Junior \\ ORCID: https://orcid.org/0000-0003-0579-3938 \\ Centro Universitário Augusto Motta, Brasil \\ E-mail: elsonnobrega@souunisuam.com.br \\ Luiz Felipe Basilio dos Santos \\ ORCID: https://orcid.org/0000-0003-2215-5540 \\ Centro Universitário Augusto Motta, Brasil \\ E-mail: Luizfelpb.santos@hotmail.com \\ Carlos Eduardo Rafael de Andrade Ferrari \\ ORCID: https://orcid.org/0000-0001-8671-7448 \\ Universidade do Porto, Portugal \\ E-mail: ceraferrari@yahoo.com.br \\ Rafael Mocarzel \\ ORCID: https://orcid.org/0000-0001-9480-826X \\ Universidade Federal do Rio de Janeiro, Brasil \\ Universidade de Vassouras, Brasil \\ Universidade do Porto, Portugal \\ E-mail: professormocarzel@gmail.com \\ Jeter Pereira de Freitas \\ ORCID: https://orcid.org/0000-0001-6540-5218 \\ Centro Universitário Augusto Motta, Brasil \\ E-mail: jeterfreitas@gmail.com \\ Marcelo José Colonna de Miranda \\ ORCID: https://orcid.org/0000-0003-1552-9153 \\ Centro Universitário Augusto Motta, Brasil \\ Universidade Estácio de Sá, Brasil \\ E-mail: marcelocolonna71@gmail.com \\ Estêvão Rios Monteiro \\ ORCID: http://orcid.org/0000-0003-1866-553x \\ Centro Universitário Augusto Motta, Brasil \\ Centro Universitário IBMR, Brasil \\ Universidade Federal do Rio de Janeiro, Brasil \\ E-mail: profestevaomonteiro@gmail.com
}

\begin{abstract}
Resumo
O objetivo do presente estudo foi revisar os efeitos da pandemia por COVID-19 sobre o nível de atividade física e sedentarismo. Foi realizado um levantamento de artigos na base de dados do Google Acadêmico, sendo incluídos artigos publicados até setembro de 2021 e usando como descritores: "Sedentarismo", "Atividade física e saúde", "Manutenção da saúde", e "COVID-19". Foram encontrados um total de 16.200 artigos na base de dados do Google Acadêmico e, após passarem pelo critério de inclusão e exclusão, foram incluídos um total de 13 artigos. Majoritariamente, os artigos avaliados nesta revisão de literatura indicam efeitos negativos no nível de atividade física e grau de sedentarismo ao longo das restrições sanitárias no COVID-19. Assim, é possível concluir que houve piora na saúde da população e que está diretamente relacionada ao baixo nível de atividade física e aumento do comportamento sedentário. Desta forma, os autores reforçam a importância de se manter uma prática de atividade física regular.
\end{abstract}

Palavras-chave: Atividade física e saúde; COVID-19; Inatividade física; Sedentarismo; Manutenção da saúde.

\section{Abstract}

The purpose of the present study was to review the effects of the COVID-19 pandemic on the level of physical activity and sedentary lifestyle. A search articles was carried out throughout Google Scholar database, including 
articles published until September 2021 and using as descriptors: 'Sedentary lifestyle', 'Physical activity and health', 'Health maintenance', and 'COVID-19'. A total of 16.200 articles were found in the Google Scholar database and, after confirmed inclusion and exclusion criteria, a total of 13 articles were included. Mostly included articles indicate negative effects on the physical activity level and sedentary lifestyle throughout COVID-19 sanitary restrictions. Thus, it is possible to conclude that the population's health worsened and that it is directly related to the low level of physical activity and increased sedentary behavior. Thus, the authors reinforce the importance of maintaining regular physical activity.

Keywords: Physical activity and health; COVID-19; Physical inactivity; Sedentary lifestyle; Health maintenance.

\section{Resumen}

El propósito del presente estudio fue revisar los efectos de la pandemia COVID-19 sobre el nivel de actividad física y el sedentarismo. Se realizó una búsqueda de artículos en la base de datos de Google Scholar, incluyendo artículos publicados hasta septiembre 2021 y utilizando como descriptores: 'Estilo de vida sedentario', 'Actividad física y salud', 'Mantenimiento de la salud' y 'COVID-19'. Se encontraron un total de 16.200 artículos en la base de datos de Google Scholar y, después de confirmar los criterios de inclusión y exclusión, se incluyeron un total de 13 artículos. En su mayoría, los artículos evaluados en esta revisión de la literatura indican efectos negativos sobre el nivel de actividad física y el grado de sedentarismo junto con restricciones sanitarias en COVID-19. Así, es posible concluir que la salud de la población empeoró y que está directamente relacionada con el bajo nivel de actividad física y el aumento del sedentarismo. Así, los autores refuerzan la importancia de mantener una actividad física regular.

Palabras clave: Actividad física y salud; COVID-19; La inactividad física; Estilo de vida sedentario; Mantenimiento de la salud.

\section{Introdução}

No final do ano de 2019, um novo coronavírus (o SARS-CoV-2) foi identificado como a causa de um conjunto de casos de pneumonia grave na cidade de Wuhan, província de Hubei, na China. A infecção do trato respiratório causada por esse vírus recebeu o nome de Coronavirus Disease (COVID-19). O SARS-CoV-2 é altamente contagioso e, por isso, desde a sua emergência o vírus rapidamente se alastrou pelo mundo tomando proporções pandêmicas (Matte et al., 2020). Segundo Cacau et al. (2020) os pacientes com COVID-19 podem desenvolver acometimentos graves durante a hospitalização tais como insuficiência respiratória, choque séptico e/ou disfunção de múltiplos órgãos levando à necessidade de oxigênio suplementar e ventilação mecânica prolongada.

A presença dos coronavírus em nossa sociedade não é recente, incluindo aqui o SARS-CoV-2. Segundo Henry (2020), o primeiro relato descrito de infecções respiratórias causada por coronavírus foi realizado por Fred Beaudette e Charles Hudson no ano de 1937 e descritos como coronavírus em 1965 (Lima, 2020). Existem diversos tipos de coronavírus descritos na literatura, destacamos aqui o SARS-CoV e o SARS-CoV-2 por serem agentes etiológicos desencadeadores da Síndrome Respiratória Aguda Grave (SDRA), sendo o segundo descrito pela primeira vez em 2019 na cidade de Wuhan na China e causador da manifestação clínica conhecida por COVID-19 (Lima, 2020).

Inicialmente, o quadro clínico de COVID-19 era considerado endêmico, mas que logo se disseminou por sua característica de fácil transmissão. A Organização Mundial da Saúde (OMS, 20201) indica que a disseminação do SARS-CoV2 a nível mundial ocorreu em pouco mais de 3 meses, onde países de todos os continentes, excluindo aqui a Antártida, registravam casos dessa nova doença. Assim, a mesma OMS decretou estado de pandemia em junho de 2020 devido a disseminação rápida e agressiva à nível mundial, ultrapassando as taxas de normalidade, mesmo sendo uma doença recente, o que reitera o sugerido por Medeiros et al. (2020) e Sousa et al. (2021) de manter o distanciamento social. A Fundação Oswaldo Cruz (FIOCRUZ, 20212) reforça em sua página oficial uma informação curiosa e conectiva. O órgão relembra que na década passada, em 2009, ocorreu caso pandêmico similar, esse menos agressivo no continente sul-americano, que foi o de gripe

\footnotetext{
${ }^{1}<$ https://www.who.int/director-general/speeches/detail/who-director-general-s-opening-remarks-at-the-media-briefing-on-covid-19---11march-2020> Acessado em 01 de dezembro de 2021 às 09 horas e 50 minutos.

$2<$ https://www.bio.fiocruz.br/index.php/br/noticias/1763-o-que-e-uma-pandemia> Acessado em 01 de dezembro de 2021 às 09 horas e 55 minutos.
} 
suína, causada pelo agente etiológico viral do H1N1. A similaridade entre as duas condições clínicas não se dá apenas pelo recorte temporal entre elas, mas sim pela similaridade do agente etiológico e do quadro clínico apresentado por ambas.

Os Muitos estudos são lançados de tempos em tempos sobre a atividade física, bem como abordagens sobre a importância dela. Com isso é possível desmembrar esta importância, seja pelo fato de estar atrelado a prática conforme o considerado ideal, ou seja, pela sua total ausência. Neste caso vale a pena ressaltar que os estudos recentes apontam para casos que a inatividade física, ou seja, a total falta de uma atividade regulada e sistêmica, aumentam exponencialmente a quantidade de agravos diretos a saúde (Gualano \& Tinucci, 2011).

Pode-se dizer que hoje, um dos grandes males da saúde pública moderna é a inatividade física. A população em sua maioria, em torno de 70\%, está já na parcela adulta, seja devido à falta de acesso por motivos econômicos, ou por uma rotina de trabalho exaustiva, não consegue atingir nem mesmo os níveis mínimos recomendados e tidos como necessários para ao menos a manutenção da base saudável (Gualano \& Tinucci, 2011).

Tendo como base a premissa acima da atividade física e remetendo esta ao ano atual, 2021, ano este que a pandemia de COVID 19 assolou e ainda assola até o presente momento a humanidade. É necessário pontuar que esta trouxe com ela tanto as causas e consequências da enfermidade como também uma maior necessidade ao longo deste tempo de permanecer em casa, aumentando assim a porcentagem da população retratada acima e reduzindo ainda mais a quantidade por si só de tempo e de tipo de atividade física realizada. (Costa et al., 2020).

Dessa forma, o objetivo do presente estudo foi revisar os efeitos da pandemia por COVID-19 sobre o nível de atividade física e sedentarismo.

\section{Metodologia}

\subsection{Tipo de estudo}

O estudo em tela é uma pesquisa de cunho de revisão de literatura (Estrela, 2018).

\subsection{Seleção dos artigos}

Houve levantamento de artigos científicos em fontes de referência como Google Acadêmico até o mês dezembro de 2021. Os seguintes termos descritores foram utilizados nas buscas e levantamento: "Sedentarismo", "Atividade física e Saúde", "Manutenção da saúde", "COVID 19". Os estudos encontrados como resultado da pesquisa realizada com estes descritores foram filtrados e selecionados com base em alguns critérios de inclusão, sendo eles: a) textos que não abordam apenas uma faixa etária restringida, b) resultados tendo como base a prática de atividade física em indivíduos com sedentarismo e comprometimentos de saúde causados pelo mesmo, c) resultados fisiológicos dentro apresentados dentro do parâmetro anteriormente citado, além de período vigente do início da pandemia até o atual e d) artigos publicados tanto em português quanto em inglês. Foram excluídos da pesquisa estudos oriundos de trabalhos acadêmicos de formação, tais como monografias, dissertações e teses, assim como artigos realizados em modelos animais.

Os envolvidos nas pesquisas selecionaram textos que mais se adequavam ao objetivo retratado e após escolher quais seriam melhores abordagens para o assunto, dentre estes, realizaram leitura na íntegra para os utilizar. As pesquisas que continham conteúdos fisiológicos e dados gráficos foram utilizadas de forma escrita, baseando o artigo aqui presente e tendo suas devidas referências apresentadas.

\section{Resultados e Discussão}

Foram encontrados um total de 47 artigos na base de dados do Google Acadêmico. Após passarem pelo critério de inclusão e exclusão, foram analisados 13 artigos no total: Fédération Internationale de Médecine Sportive (1998), Amorim et 
al. (2002), Gualano e Tinucci (2011), Bezerra et al. (2020), Costa et al. (2020), Gomes et al. (2020), Guimarães (2020), Malta et al. (2020), Sá et al. (2020), Brito et al. (2021), Carvalho et al. (2021), Gomes et al (2021) Pitanga et al. (2021). O Quadro 1 traz a síntese das principais informações extraídas dos estudos.

Quadro 1 - Descrição dos estudos selecionados.

\begin{tabular}{|c|c|c|c|c|c|c|}
\hline $\begin{array}{l}\text { Autores e } \\
\quad \text { ano }\end{array}$ & População & Intervenção & Comparação & Protocolo & Resultado & Conclusão \\
\hline $\begin{array}{l}\text { Fédération } \\
\text { Internation } \\
\text { ale de } \\
\text { Médecine } \\
\text { Sportive } \\
\text { (1998) }\end{array}$ & $\begin{array}{l}\text { Não } \\
\text { Especificado. }\end{array}$ & $\begin{array}{l}\text { Apanhado teórico } \\
\text { sobre atividade } \\
\text { física. }\end{array}$ & Não Especificado. & $\begin{array}{l}\text { Posicionamento científico } \\
\text { aprovado. }\end{array}$ & $\begin{array}{l}\text { Dados sobre diversos impactos a } \\
\text { saúde corpórea dos indivíduos. }\end{array}$ & $\begin{array}{l}\text { O sedentarismo e a ausência de } \\
\text { adaptações induzidas pelo } \\
\text { exercício regular reduzem as } \\
\text { reservas fisiológicas do corpo, } \\
\text { o que acarreta vários riscos } \\
\text { para a saúde e a capacidade } \\
\text { física. }\end{array}$ \\
\hline $\begin{array}{l}\text { Amorim et } \\
\text { al. }(2002)\end{array}$ & 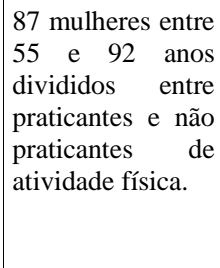 & $\begin{array}{|lr|}\text { Avaliaram-se } & \\
\text { características } & \text { da } \\
\text { aptidão } & \text { físicas } \\
\text { inerentes } & \text { à } \\
\text { manutenção } & \text { da } \\
\text { capacidade } & \\
\text { funcional. } & \end{array}$ & 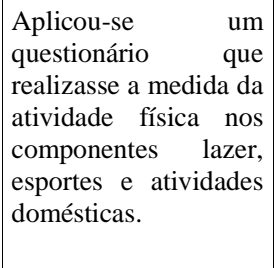 & $\begin{array}{l}\text { Força de membros } \\
\text { superiores, dinamometria } \\
\text { dorsal e de membros } \\
\text { inferiores, amplitude da } \\
\text { passada em marcha, teste de } \\
\text { sentar-se e alcançar, tempo } \\
\text { de reação e questionário para } \\
\text { idosos. }\end{array}$ & $\begin{array}{l}\text { Grupo ativo possui maior força } \\
\text { muscular, flexibilidade, nenhum } \\
\text { ganho relevante a que se refere } \\
\text { ao tempo de reação. }\end{array}$ & $\begin{array}{l}\text { O grupo ativo apresenta uma } \\
\text { melhor capacidade funcional e } \\
\text { um estilo de vida mais ativo, } \\
\text { quando consideradas as } \\
\text { atividades domésticas, } \\
\text { esportivas e de lazer, em } \\
\begin{array}{l}\text { comparação com o grupo } \\
\text { sedentário. }\end{array}\end{array}$ \\
\hline $\begin{array}{l}\text { Gualano e } \\
\text { Tinucci } \\
\text { (2011) }\end{array}$ & $\begin{array}{l}\text { Não } \\
\text { Especificado. }\end{array}$ & $\begin{array}{l}\text { Apanhado teórico } \\
\text { sobre atividade } \\
\text { física. }\end{array}$ & $\begin{array}{l}\text { Dados estatísticos de } \\
\text { diversas idades } \\
\text { referentes ao impacto } \\
\text { da pandemia. }\end{array}$ & Levantamento bibliográfico. & $\begin{array}{l}\text { O exercício físico é uma } \\
\text { ferramenta barata, segura, não } \\
\text { patenteável e, quando prescrita } \\
\text { de maneira correta, põe fim à } \\
\text { necessidade de uma vasta gama } \\
\text { de medicamentos }\end{array}$ & $\begin{array}{l}\text { Cabe ao profissional de } \\
\text { Educação Física capacitar-se } \\
\text { afim de prescrever o exercício } \\
\text { com eficácia e segurança a } \\
\text { toda população. }\end{array}$ \\
\hline $\begin{array}{l}\text { Bezerra et } \\
\text { al. }(2020)\end{array}$ & $\begin{array}{l}\text { Não } \\
\text { Especificado. }\end{array}$ & $\begin{array}{lr}\text { Trata-se de } & \text { um } \\
\text { estudo transversal } \\
\text { realizado a } & \text { partir } \\
\text { de } & \text { um } \\
\text { questionário } & \text { com } \\
\text { dezessete } & \\
\text { perguntas } & \\
\text { objetivas } & \text { no } \\
\text { formato } & \text { de } \\
\text { pesquisa } & \text { de } \\
\text { opinião. } & \end{array}$ & $\begin{array}{l}\text { O instrumento de } \\
\text { coleta foi construído } \\
\text { nar plataforma } \\
\text { Formulários Google e } \\
\text { divulgado via internet, } \\
\text { através dos aplicativos } \\
\text { e redes sociais. }\end{array}$ & $\begin{array}{l}\text { Questões referentes ao perfil } \\
\text { socioeconômico dos } \\
\text { respondentes, isolamento e } \\
\text { ao impacto dele na vida das } \\
\text { pessoas, condições de } \\
\text { habitabilidade no isolamento } \\
\text { e expectativa das pessoas em } \\
\text { relação ao tempo máximo } \\
\text { que acreditam suportar o } \\
\text { isolamento. }\end{array}$ & $\begin{array}{l}\text { Os resultados gerais revelam que } \\
\text { estresse é apontado como uma } \\
\text { das principais consequências do } \\
\text { isolamento social ocasionando } \\
\text { alterações no sono. Além do } \\
\text { desafio da prática de atividade } \\
\text { física. }\end{array}$ & $\begin{array}{l}\text { É necessário o melhor } \\
\text { entendimento de como a } \\
\text { estratégia de isolamento social } \\
\text { é percebida pela sociedade e } \\
\text { quais os impactos dessa } \\
\text { estratégia na vida das pessoas. }\end{array}$ \\
\hline $\begin{array}{l}\text { Costa. et } \\
\text { al. }(2020)\end{array}$ & $\begin{array}{l}\text { Sujeitos adultos } \\
\text { (maiores de } 18 \\
\text { anos de idade), de } \\
\text { ambos os sexos e } \\
\text { que reportaram } \\
\text { residir em } \\
\text { municípios das } \\
\text { cinco regiões do } \\
\text { Brasil. }\end{array}$ & $\begin{array}{lr}\text { Foi aplicado } & \text { o } \\
\text { teste } & \\
\text { Kolmogorov- } & \\
\text { Smirnov } & \text { para } \\
\text { avaliar } & \mathrm{a} \\
\text { normalidade } & \text { dos } \\
\text { dados. } & \\
& \end{array}$ & 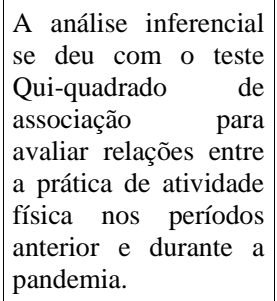 & $\begin{array}{l}\text { Sete questões relacionadas a } \\
\text { aspectos sociodemográficos, } \\
\text { (zona metropolitana ou } \\
\text { interior) e de um. E mais } \\
\text { questões considerando o } \\
\text { período de recordatório: } \\
\text { antes da pandemia e durante } \\
\text { a pandemia. }\end{array}$ & $\begin{array}{l}\text { Em relação à prática de atividade } \\
\text { física, observa-se que antes da } \\
\text { pandemia a maioria dos } \\
\text { participantes } 65,5 \% \text { e houve um } \\
\text { aumento do percentual para } \\
80,1 \% \text {. }\end{array}$ & $\begin{array}{l}\text { Desta forma, o estudo traz } \\
\text { implicações práticas para que } \\
\text { sejam pensadas formas de } \\
\text { incentivar a prática de } \\
\text { atividade física durante } \\
\text { períodos de distanciamento } \\
\text { social. }\end{array}$ \\
\hline $\begin{array}{l}\text { Gomes et } \\
\text { al. }(2020)\end{array}$ & $\begin{array}{l}\text { Indivíduos } \\
\text { adultos maiores } \\
\text { de } 18 \text { anos } \text { e } \\
\text { residentes no } \\
\text { Brasil. }\end{array}$ & $\begin{array}{l}\text { Amostragem em } \\
\text { cadeia. }\end{array}$ & \begin{tabular}{|lr} 
Por meio de & pessoas \\
convidadas & que \\
geravam & mais \\
convites. & \\
& \\
&
\end{tabular} & $\begin{array}{l}\text { As questões referem-se a } \\
\text { adesão às medidas de } \\
\text { restrição social, alterações } \\
\text { nos estilos de vida (hábito de } \\
\text { fumar; consumo de bebida } \\
\text { alcoólica, hábito alimentar e } \\
\text { prática de atividade física) e } \\
\text { no estado de ânimo. }\end{array}$ & $\begin{array}{l}\text { Em relação ao estado de ânimo, o } \\
\text { estudo revelou que } 41,2 \%, 41,3 \% \\
\text { e } 35,2 \% \text { dos brasileiros se } \\
\text { sentiram isolados, ansiosos, e } \\
\text { tristes ou deprimidos por muitas } \\
\text { vezes, respectivamente, sendo as } \\
\text { prevalências maiores nas } \\
\text { mulheres comparadas aos } \\
\text { homens. }\end{array}$ & $\begin{array}{l}\text { Os resultados devem apoiar } \\
\text { profissionais de saúde e } \\
\text { gestores na priorização de } \\
\text { estratégias de promoção de } \\
\text { saúde durante e após a } \\
\text { pandemia. }\end{array}$ \\
\hline $\begin{array}{l}\text { Guimarães } \\
(2020)\end{array}$ & $\begin{array}{l}\text { Não } \\
\text { Especificado. }\end{array}$ & $\begin{array}{l}\text { Apanhado teórico } \\
\text { sobre atividade } \\
\text { física. }\end{array}$ & $\begin{array}{l}\text { Dados estatísticos de } \\
\text { diversas idades } \\
\text { referentes ao impacto } \\
\text { da pandemia. }\end{array}$ & Levantamento bibliográfico. & $\begin{array}{l}\text { O distanciamento social imposto } \\
\text { pela epidemia de COVID-19 } \\
\text { acarreta dificuldades rara } \\
\text { realização das estratégias } \\
\text { enfrentamento e estimula as } \\
\text { práticas que podem desencadear } \\
\text { ou agravar o excesso de peso, } \\
\text { sendo potencialmenter um } \\
\text { complicador da epidemia de } \\
\text { obesidade cujos reais impactos }\end{array}$ & $\begin{array}{l}\text { As modificações nos hábitos } \\
\text { de vida no que tange à } \\
\text { restrição de atividades, } \\
\text { alterações da dinâmica } \\
\text { alimentar e aumento do tempo } \\
\text { de utilização de eletrônicos, } \\
\text { contribuem para um balanço } \\
\text { energético positivo e, } \\
\text { consequentemente, para maior } \\
\text { possibilidade de surgimento ou }\end{array}$ \\
\hline
\end{tabular}




\begin{tabular}{|c|c|c|c|c|c|c|}
\hline & & & & & serão percebidos posteriormente. & $\begin{array}{l}\text { agravamento de sobrepeso e } \\
\text { obesidade em crianças e } \\
\text { adolescentes. }\end{array}$ \\
\hline $\begin{array}{l}\text { Malta et al. } \\
(2020)\end{array}$ & $\begin{array}{lll}\text { Brasileiros de } & 18 \\
\text { a maiores } & \text { de } & 60 \\
\text { anos. } & & \end{array}$ & $\begin{array}{l}\text { Amostragem em } \\
\text { cadeia. }\end{array}$ & $\begin{array}{lr}\text { Por meio de } & \text { pessoas } \\
\text { convidadas } & \text { que } \\
\text { geravam } & \text { mais } \\
\text { convites. } & \end{array}$ & $\begin{array}{l}\text { Levantamento acerca da } \\
\text { mudança na vida dos adultos } \\
\text { brasileiros. }\end{array}$ & 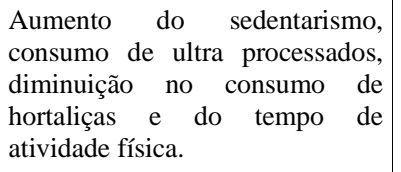 & $\begin{array}{l}\text { Faz-se necessária a ação de } \\
\text { políticas públicas, promoção } \\
\text { de saúde e ampliação da } \\
\text { atividade primária. }\end{array}$ \\
\hline $\begin{array}{l}\text { Sá et al. } \\
(2020)\end{array}$ & $\begin{array}{l}\text { Famílias com } \\
\text { crianças de até } 13 \\
\text { anos de idade. }\end{array}$ & $\begin{array}{l}\text { Pesquisa on-line } \\
\text { anônima. }\end{array}$ & $\begin{array}{l}\text { Cada família relatou o } \\
\text { tempo diário em que } \\
\text { cada criança esteve } \\
\text { envolvida. }\end{array}$ & $\begin{array}{l}\text { Atividade } \begin{array}{l}\text { sedentária } \\
\text { (somatório de atividades }\end{array} \\
\text { intelectuais, tempo lúdico de } \\
\text { tela, brincar sem AF) e AF } \\
\text { (somatório do brincar com } \\
\text { AF e da AF. }\end{array}$ & $\begin{array}{l}\text { Os principais achados indicam } \\
\text { que a maioria dos pais considera } \\
\text { que houve redução no tempo em } \\
\text { que as crianças passam } \\
\text { praticando AF e aumento do } \\
\text { tempo lúdico de tela e das } \\
\text { atividades em família. }\end{array}$ & $\begin{array}{l}\text { As rotinas domiciliares das } \\
\text { famílias durante o período de } \\
\text { distanciamento } \\
\text { decorrente da pandemia de } \\
\text { COVID-19 confirmam a } \\
\text { tendência geral decrescente do } \\
\text { tempo de AF na infância. }\end{array}$ \\
\hline $\begin{array}{l}\text { Brito et al. } \\
(2021)\end{array}$ & $\begin{array}{l}\text { Jovens, Adultos e } \\
\text { Idosos de idades } \\
\text { não restritas. }\end{array}$ & $\begin{array}{l}\text { Trata-se de um } \\
\text { estudo realizado } \\
\text { por meio de } \\
\text { questionário. }\end{array}$ & $\begin{array}{l}\text { Foi agrupado em três } \\
\text { categorias, adultos } \\
\text { jovens (18-40 anos), } \\
\text { adultos de meia-idade } \\
(41-60 \text { anos) e idosos } \\
(>60 \text { anos). }\end{array}$ & $\begin{array}{l}\text { O questionário foi aplicado } \\
\text { via Forms e depois de } \\
\text { efetivado sua aplicação e } \\
\text { encerrado o prazo, houve a } \\
\text { coleta de dados }\end{array}$ & $\begin{array}{l}\text { A análise } \quad \text { evidenciou a } \\
\text { importância de ter um } \\
\text { profissional de educação física } \\
\text { ministrando aulas para a } \\
\text { manutenção da atividade física. }\end{array}$ & $\begin{array}{l}\text { Concluiu-se que o isolamento } \\
\text { social promoveu maior } \\
\text { impacto negativo sobre os } \\
\text { hábitos de atividade física no } \\
\text { grupo mais jovem (adultos } \\
\text { jovens), que utilizou mais } \\
\text { tempo em atividades } \\
\text { sedentárias. }\end{array}$ \\
\hline $\begin{array}{l}\text { Carvalho, } \\
(2021)\end{array}$ & $\begin{array}{l}\text { Não } \\
\text { Especificado. }\end{array}$ & $\begin{array}{l}\text { Apanhado teórico } \\
\text { sobre atividade } \\
\text { física. }\end{array}$ & $\begin{array}{l}\text { Dados estatísticos de } \\
\text { diversas idades } \\
\text { referentes ao impacto } \\
\text { da pandemia. }\end{array}$ & Levantamento bibliográfico. & $\begin{array}{l}\text { Há necessidade de correlação } \\
\text { entre atividade física e saúde } \\
\text { básica. }\end{array}$ & $\begin{array}{l}\text { Ampliação da atividade física } \\
\text { em prol da redução da } \\
\text { mortalidade. }\end{array}$ \\
\hline $\begin{array}{l}\text { Gomes et } \\
\text { al. }(2021)\end{array}$ & $\begin{array}{l}\text { Adolescentes } \\
\text { brasileiros de } 12 \\
\text { a } 17 \text { anos. }\end{array}$ & $\begin{array}{l}\text { Questionário via } \\
\text { web e smartfones. }\end{array}$ & $\begin{array}{l}\text { Questões sobre } \\
\text { condições } \\
\text { sociodemográficas e } \\
\text { mudanças no estilo de } \\
\text { vida. }\end{array}$ & $\begin{array}{l}\text { Pesquisadores repassavam as } \\
\text { pesquisas a outros que } \\
\text { divulgavam para a faixa } \\
\text { etária pretendida. }\end{array}$ & $\begin{array}{l}\text { Aumento da má alimentação, do } \\
\text { sedentarismo, do fumo e redução } \\
\text { da atividade física. }\end{array}$ & $\begin{array}{l}\text { Necessário de reforço dos } \\
\text { familiares e das políticas } \\
\text { públicas para com estes } \\
\text { adolescentes. }\end{array}$ \\
\hline $\begin{array}{l}\text { Pitanga et } \\
\text { al. }(2021)\end{array}$ & $\begin{array}{lll}\text { Dados } & \text { de } \\
\text { residentes } & \text { em } & 26 \\
\text { capitais } & & \\
\text { brasileiras } & \text { e } & \text { no } \\
\text { Distrito } & \text { Federal } \\
\text { sem distinção } & \text { de } \\
\text { idade. } & \end{array}$ & $\begin{array}{l}\text { Estudo ecológico } \\
\text { transversal } \\
\text { observacional. }\end{array}$ & $\begin{array}{l}\text { Dados retirados } \\
\text { diretamente do SUS } \\
\text { quanto a COVID-19. }\end{array}$ & $\begin{array}{l}\text { Foram analisados dados de } \\
\text { prevalência de atividade } \\
\text { física no lazer (AFL), óbitos } \\
\text { acumulados, letalidade da } \\
\text { doença e taxa de mortalidade } \\
\text { por Covid-19. }\end{array}$ & $\begin{array}{l}\text { Os resultados demonstram a } \\
\text { existência de associação inversa, } \\
\text { mesmo após ajuste para variáveis } \\
\text { de confusão entre AFTL, óbitos } \\
\text { acumulados e letalidade da } \\
\text { doença. }\end{array}$ & $\begin{array}{l}\text { Os resultados do presente } \\
\text { estudo nos levam a acreditar } \\
\text { que a prática de atividade } \\
\text { física no lazer pode ser um } \\
\text { importante aliado no } \\
\text { enfrentamento de Covid-19. }\end{array}$ \\
\hline
\end{tabular}

Legenda: AF - Atividade Física / AFL e AFTL - Atividade Física e Lazer. Fonte: Autores.

O objetivo do presente estudo foi revisar os efeitos da pandemia por COVID-19 sobre o nível de atividade física e sedentarismo. Inicialmente, é possível evidenciar que tanto a inatividade física quanto o sedentarismo parecem serem causadores e/ou facilitadores das complicações de diversas condições clínicas ao longo do processo de pandemia. É possível citar condições clínicas como pressão alta, diabetes, obesidade entre outras, que possuem seu surgimento ou agravamento decorrente deste cenário. Vale ressaltar ainda que está problemática abrange diversas faixas etárias, sejam elas, crianças, adolescentes, adultos e idosos (Gualano \& Tinucci, 2011)

Dentro deste aspecto de inatividade e mazelas, se apresentou a pandemia decorrente da COVID-19 e com ela a restrição da quarentena. Este episódio influenciou diretamente na saúde da população, uma vez que pessoas foram mantidas privadas de suas atividades físicas diárias, as academias, que hoje são o grande foco e representação da atividade física seja para saúde ou estética, teve seu acesso proibido por muitos meses até ser novamente liberada, com diversas restrições. Houve acesso a comida de forma não regulada e estresse emocionais diversos em decorrência da própria quarentena, da perda dos postos de trabalho, da ausência ou perda de entes queridos entre outros (Malta et al., 2021).

Tendo como análise este panorama, a rotina desta população atingida psicologicamente por estresse de ter seu emprego diário retirado ou ainda, realizado de forma remota, se aliou a falta ou menor realização de atividade física, aumento do tempo sedentário de forma geral, bem como alta ingestão de alimentos ultra processados, horários irregulares de sono decorrente a ansiedade e trabalho remoto desorganizado. Isto ainda pode ser atrelado a condição brasileira de acesso a saúde e 
a condição financeira das famílias que já eram bem precárias. Acabou por acelerar diretamente as condições preexistentes e ainda criar condições que antes não eram presentes em certos indivíduos, decorrentes deste episódio complexo de sedentarismo aliado a mudança bruta de hábitos (Malta et al., 2021).

Agora que a pandemia avança para seu término junto com o relaxamento das restrições, a população tenta normalizar sua rotina de modo geral, contudo, suas consequências sedentárias diretas estarão presentes em breve, uma vez que a qualidade de vida da população de modo geral piorou, principalmente no que se refere as condições econômicas das famílias brasileiras, e a falta de atividade. A população adulta sofreu diretamente o golpe desta inatividade e em modo geral houve piora no estilo de vida. Ao ponto de estimar cerca de agravamento de indicadores e maiores índices de mortalidades decorridos a doenças geradas por consequência deste panorama como a obesidade, por exemplo, aos longos dos anos que virão (Guimarães, 2020)

Os focos de atenção nesta população devem ser diversos, já que no geral o aspecto fisiológico e psicológico foi amplamente afetado. É fato que o sedentarismo está aliado muitas vezes a obesidade por consequência, mas outros fatores como o sistema cardíaco, respiratório e o metabolismo em si podem ser prejudicados sem que uma obesidade severa seja perceptível logo em um primeiro momento. (Fédération Internationale De Médecine Sportive, 1998).

A inatividade física causa um efeito devastador ao efeito de bombear do nosso miocárdio. Isto acaba resultando a frente, em uma oxigenação insuficiente para o corpo, causando isquemias por exemplo. Já quanto ao sistema respiratório, é possível mencionar que a inatividade causa graves consequências a musculatura ventilatória, prejudicando a capacidade respiratória tanto em repouso quanto em movimento. Por fim, o sistema metabólico, normalmente norteado pela tipificação dos componentes do colesterol ditos bom e ruim, e que somando - se a isto, há algum tempo já é conhecido que o sedentarismo, bem como obesidade está associado ao aumento do colesterol (Fédération Internationale De Médecine Sportive, 1998)

Podemos citar em especial o LDL (lipoproteína de baixa densidade) esta, por si só aumenta a chance de diversos outros fatores preocupantes como o entupimento de artérias, bem como ocorrências degenerativas das mesmas. Entretanto, ainda é possível observar, que comprometimentos progressivos e extensos ocorrem em toda a fisiologia do ser em questão que este inativo fisicamente, de forma resumida, podemos observar que sua termorregulação, atividade fagocitária, resistência a substâncias tóxicas, tolerância a hipoxia e a hipoidratação, assim como também a sua psique estão em constante declínio. (Fédération Internationale De Médecine Sportive, 1998)

Sendo assim se faz necessário, de forma progressiva e o quanto antes, que políticas públicas sejam implantadas para promover saúde, principalmente no que se refere ao setor primário da saúde, tendo amplo foco aliado a não só se consultar e checar seu estado fisiológico como também divulgar intensamente a importância de atividades físicas ao menos mínimas e necessárias para o corpo, bem como recomendado pela Organização mundial da Saúde (Malta et al., 2021).

\section{Considerações Finais}

É possível concluir que apesar de se tratar de um tema complexo, fica claro que a inatividade física e sedentarismo já existe com força na população mundial, acarretando diversas doenças silenciosas e que trazem consigo um elevado índice de mortalidade. É fato de que existem fatores como os socioeconômicos, educacionais e a própria reclusão da pandemia que acabaram por acelerar ainda mais este processo, contudo fica nítido que o educador físico e sua classe como um todo, precisa o quanto antes, aliado de políticas públicas redefinir as prioridades de ensino e educar a população de suas regiões a fim de promover saúde em larga escala e evitar através da mesma que este indivíduo se trate através de ingestões de químicos ou até mesmo venha a evoluir para casos graves que levem a sequelas ou a morte.

Mesmo que a literatura prévia (Polero et al., 2020; Scartoni et al., 2020; Silveira et al., 2021; Vancini et al., 2021) sugira efeitos positivos no sistema imune de praticantes de exercício físico, ela não consegue sustentar esses mesmos efeitos contra os sinais e sintomas da COVID-19. Ademais, o efeito imunoprotetor do exercício físico requer uma sistematização e 
continuidade da atividade. Pensando nisso, os autores deste presente artigo reforçam a necessidade de melhores investigações acerca da COVID-19, haja visto a real necessidade de compreendê-la. Por fim, segue a sugestão de estudos longitudinais observacionais em pacientes fisicamente ativos e fisicamente inativos durante após diagnóstico clínico de COVID-19.

\section{Referências}

Amorim, P. R. S., Miranda, M., Chiapeta, S. M. V., et al. (2002). Estilo de vida ativo ou sedentário: Impacto sobre a capacidade funcional. Rev Bras Cienc Esporte, 23 (3), 49-63.

Bezerra, A. C. V., Silva, C. E. M., Soares, F. R. G., et al. (2020). Fatores associados ao comportamento da população durante o isolamento social na pandemia de Covid-19. Rev Ciência e Saúde Coletiva, 25, 2411-2421.

Brito, L. M. S., Leite, N., Lima, V. A., et al. (2021). Atividade Física, hábitos alimentares e sono durante o isolamento social: do jovem ao idoso. Rev Bras Med Esporte, 27 (1), 21-25.

Cacau, L. de A. P., Mesquita, R., Furlanetto, K. C., Borges, D. L. S., Forgiarini Júnior, L. A., et al. (2020). Avaliação e intervenção para a reabilitação cardiopulmonar de pacientes recuperados da COVID-19. ASSOBRAFIR Ciência, 11 (1), 183-193.

Carvalho, F. F. B., Freitas, D. D., \& Akerman, M. (2021). O “novo normal” na atividade física e saúde: pandemias e uberização? Movimento, $27,27022$.

Costa, C. L. A. C., Costa, T. M., Filho, V. C. B., et al. (2020). Influência do distanciamento social no nível de atividade física durante a pandemia do Covid19. Rev Bras Ativ Fís e Saúde, 25, 1-6.

Estrela, C. (2018). Metodologia Científica: Ciência, Ensino, Pesquisa. Editora Artes Médicas.

Fédération Intenationale de Médecine Sportive - Posicionamento Oficial. (1998). A atividade física aumenta os fatores de risco para a saúde e a capacidade física. Rev Bras Med Esporte, 4 (2), 69-70.

Gomes, C. S., Malta, D. C., Cardoso, L. S. M., et al. (2021). A pandemia de Covid-19 e mudanças nos estilos de vida dos adolescentes brasileiros. Rev Bras Epidemiol, 24, 1-13.

Gomes, C. S., Malta, D. C., Silva, A, G., et al. (2020). Distanciamento social, sentimento de tristeza e estilos de vida da população brasileira durante a pandemia de Covid-19. Rev Saúde Debate, 44 (4), 177-190.

Gualano, B., \& Tinucci T. (2011). Sedentarismo, exercício físico e doenças crônicas. Rev Bras Educ Fís Esporte, $25,37-43$.

Guimarães, P. R. (2020). A pandemia de COVID-19 e suas repercussões na epidemia da obesidade de crianças e adolescentes. Rev Elet Ac Saúde, 12, 1-8.

Henry, R. Etymologia: Coronavirus (2020). Emerg Infect Dis, 26, p. 1027.

Lima, C. M. A. O. (2020). Informações sobre o novo coronavírus (COVID-19). Radiol Bras, 53, p. 5-6.

Malta, D. C., Gomes, C. S., Barros, M. B. A., et al. (2020). Doenças crônicas não transmissíveis e mudanças nos estilos de vida durante a pandemia de Covid19 no Brasil. Rev Bras Epidemiol, 24, 1-15.

Matte, D. L., Mesquita, R., Furlanetto, K. C., \& Karsten, M. (2020). ASSOBRAFIR reforça sua missão no enfrentamento à pandemia da COVID-19. ASSOBRAFIR Ciência, 11 (1), 11-13.

Medeiros, A. Y. B. B. V. de, Pereira, E. R., Silva, R. M. C. R. A., \& Dias, F. A. Psychological phases and meaning of life in times of social isolation due the COVID-19 pandemic a reflection in the light of Viktor Frankl. Research, Society and Development, 9(5), e122953331, 10.33448/rsd-v9i5.3331. https://rsdjournal.org/index.php/rsd/article/view/3331.

Pitanga, F. J. G., Beck, C. C., Pitanga, C. P. S., et al. (2021). Physical activity and mortality due to COVID-19in the Brazilian capitals: an ecological analysis. Rev Bras Med Esporte, 27 (6), 573-577.

Polero, P., Rebollo-Seco, C., Adsuar, J., Pérez-Gómez, J., Rojo-Ramos, J., Manzano-Redondo, F., Garcia-Gordillo, M. A., \& Carlos-Vivas, J. (2020). Physical activity recommendations during COVID-19: narrative review. Int J Environ Res Public Health, 18, 65.

Scartoni FR, Sant'Ana LO, Murillo-Rodriguez E, Yamamoto T, Imperatori C, Budde H, Vianna JM and Machado S (2020). Physical Exercise and Immune System in the Elderly: Implications and Importance in COVID-19 Pandemic Period. Front. Psychol. 11:593903. 10.3389/fpsyg.2020.593903

Sá, C. S. C., Cordovil, R., Luz, C., et al. (2020). Distanciamento social COVID - 19 no Brasil: efeitos sobre a rotina de atividade física de famílias com crianças. Rev Paul Pediatra, 39, 1-8.

Silveira, M. P., Fagundes, K. K. S., Bizutti, M. R., Starck, E., Rossi, R. C., \& Resende e Silva, D. T. (2021). Physical exercise as a tool to help the immune system against COVID-19: an integrative review of the current literature. Clin Exp Med, 21, 15-28.

Sousa, G. O., Sales, B. N., Rodrigues, A. M. X., Rocha, G. M. de M., \& Oliveira, G. A. L. de. Epidemiological evolution of COVID-19 in Brazil and worldwide. Research, Society and Development, [S. 1.], v. 9, n. 7, p. e630974653, 2020. DOI: 10.33448/rsd-v9i7.4653. Disponível em: https://rsdjournal.org/index.php/rsd/article/view/4653. Acesso em: 1 dec. 2021.

Vancini, R. L., Andrade, M. S., Viana, R. B., Nikolaidis, P. T., Knechtle, B., Campanhero, C. R. V., Almeida, A. A., Gentil, P., \& Lira, C. A. B. (2021). Physical exercise and COVID-19 pandemic in PubMed: two months of dynamic and one year of original scientific production. Sports Med Health Sci, 3, 8092. 